\title{
Mapping the circumnuclear dust in nearby AGN with MIDI
}

\author{
Konrad R. W. Tristram, ${ }^{1}$ Klaus Meisenheimer, ${ }^{1}$ Walter Jaffe ${ }^{2}$ \\ and William D. Cotton ${ }^{3}$ \\ ${ }^{1}$ Max-Planck-Institut für Astronomie, Königstuhl 17, 69117 Heidelberg, Germany \\ 2 Sterrewacht Leiden, Postbus 9513, NL-2300 RA Leiden, The Netherlands \\ ${ }^{3}$ NRAO, 520 Edgemont Road, Charlottesville, VA 22903-2475, USA \\ email: tristram@mpia.de
}

\begin{abstract}
We observed four nearby AGN with MIDI at the VLTI to investigate the mid-infrared emission from these sources. With our measurements we resolve the dusty structure around the nucleus of the Circinus galaxy. We find two dust components: a hot, small elongated structure with a size of $0.4 \mathrm{pc}$ and a cooler, almost round component with a size of $1.9 \mathrm{pc}$. We interpret the emission to be originating in a geometrically thick dusty torus oriented perpendicular to the ionisation cone. Hence our finding nicely confirms the unified picture. We also observed the nucleus of Centaurus A and find that $70 \%$ of the mid infrared flux originates from an unresolved source with a size of less than $0.2 \mathrm{pc}$. In this case, the majority of the emission comes from a synchrotron source at the base of the radio jet. Two further galaxies, Mrk 1239 (Seyfert 1) and MCG -05-23-016 (Seyfert 2), also show unresolved mid infrared sources limiting the size of the dust distribution to less than 5 and 2 pc respectively.
\end{abstract}

Keywords. Galaxies: active - galaxies: nuclei - galaxies: Seyfert - radiation mechanisms: thermal - techniques: interferometric

\section{Introduction}

In the "unified scheme", the standard model for Active Galactic Nuclei (AGN), a hot accretion disk around a supermassive black hole is assumed to be surrounded by a geometrically thick distribution of gas and dust, the dusty torus. Originally, all arguments for the existence of such tori were based on theoretical considerations and on indirect observational evidence such as the spectral energy distributions (SED) of AGN. The torus is thought to give a simple explanation for the observed dichotomy between Seyfert 1 and Seyfert 2 galaxies: type 1 objects are seen face on allowing us to directly see the central engine, while type 2 objects are oriented edge on so that the dust in the torus blocks the line of sight towards the very centre.

There is hardly any direct evidence for this scenario due to the lack of angular resolution: even for the nearest AGN the expected structures are too small (on the order of a parsec and hence smaller than 100 mas) to be resolved by single dish telescopes in the mid-infrared (MIR). Only interferometry provides the necessary angular resolution. Indeed first observations of NGC 1068 with the MID-infrared Instrument (MIDI) at the Very Large Telescope Interferometer (VLTI) of the European Southern Observatory (ESO) on Cerro Paranal were successful to resolve the circumnuclear MIR emission of this prototype Seyfert 2 galaxy, confirming the picture of the unified scheme (Jaffe et al. 2004). Since this first success, we have continued our high-resolution study of nearby AGN with MIDI, combining the light from two of the $8 \mathrm{~m}$ unit telescopes (UTs) of the VLTI. All observations were performed using guaranteed time observations (GTO). 

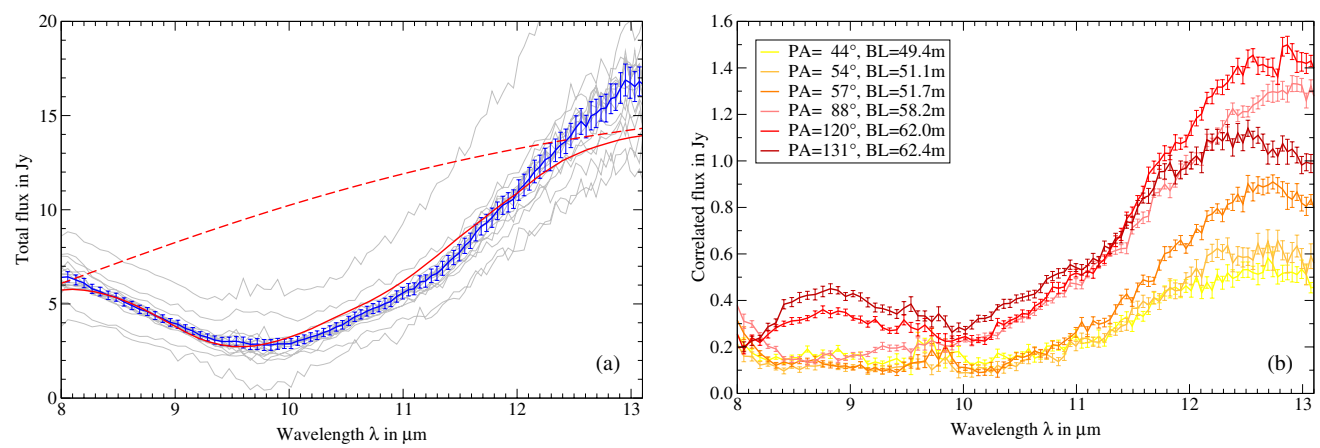

Figure 1. (a) Averaged total flux of the Circinus nucleus in the $\mathrm{N}$ band (blue) and total flux of the model (red) with silicate absorption (continuous) and without silicate absorption (dashed). (b) Correlated fluxes as observed during the night of 2005-02-28. Mainly the baseline angle changed, while the projected baseline length stayed roughly the same. From the dramatic change in the correlated fluxes, direct implications on the morphology can be derived: the source is considerably more extended in the direction of $P A \sim 50^{\circ}$ than in the direction of $P A \sim 130^{\circ}$.

\section{Circinus}

The Circinus galaxy is a highly inclined $\left(\sim 65^{\circ}\right) \mathrm{SA}(\mathrm{s}) \mathrm{b}$ galaxy harbouring a Seyfert type 2 active nucleus as well as a circumnuclear starburst. At about $4 \mathrm{Mpc}$ distance $\left(1^{\prime \prime} \sim 20\right.$ pc, Freeman et al. 1977$)$, the galaxy is among the nearest AGN and hence it is an ideal object to study the nuclear region of an active galaxy.

The interferometric data obtained on Circinus constitute the most extensive infrared interferometric data of any extragalactic source collected so far. We were able to reconstruct a total of 21 visibility points from the data. Figure 1a shows the calibrated total flux $F_{t o t}$ of Circinus (thick blue line). The broad absorption feature dominating most of the spectrum is due to silicate absorption in Circinus. We see no evidence for any line emission or emission of Polycyclic Aromatic Hydrocarbons (PAH), as observed at greater distances from the nucleus (e.g. by Roche et al. 2006).

For all baselines observed, the correlated flux $F_{\text {cor }}$ is much smaller than the total flux $\left(F_{\text {cor }} \ll F_{t o t}\right)$, indicating that the emission region is well resolved with our interferometric resolution of $\lambda / 2 B \leqslant 40$ mas. As an example, the correlated fluxes obtained on February $28^{t h}, 2005$, are shown in figure 1b. During this night, the projected baseline length only increased by a minor factor from 50 to $60 \mathrm{~m}$, while the position angle underwent a major change from 44 to $131^{\circ}$. An increase of the correlated flux can be clearly observed while the position angle increases. We directly interpret this as a change of size of the emitting source: at position angles of 44 to $57^{\circ}$ the correlated flux is low, i.e. the emission is extended, while at angles of 120 and $131^{\circ}$ the correlated flux is higher, i.e. the emission is less resolved. This is a direct and completely model independent evidence for an elongated dust structure perpendicular to the ionisation cone and outflow in Circinus (position angle $-50^{\circ}$ and opening angle $\sim 90^{\circ}$ ).

Even though the coverage is high for infrared interferometry, it is insufficient to attempt any image reconstruction. Therefore we used a simple model consisting of two black body Gaussian emitters with silicate absorption to explain the data. We find the silicate absorption profile for interstellar dust from Kemper et al. (2004) to be sufficient to describe the absorption feature we observe with MIDI, both for the total flux as well as for the correlated fluxes. The two component black body model has 10 free parameters, which are listed in figure 2 with their best fit values. The figure also contains a sketch of the best fit model. 


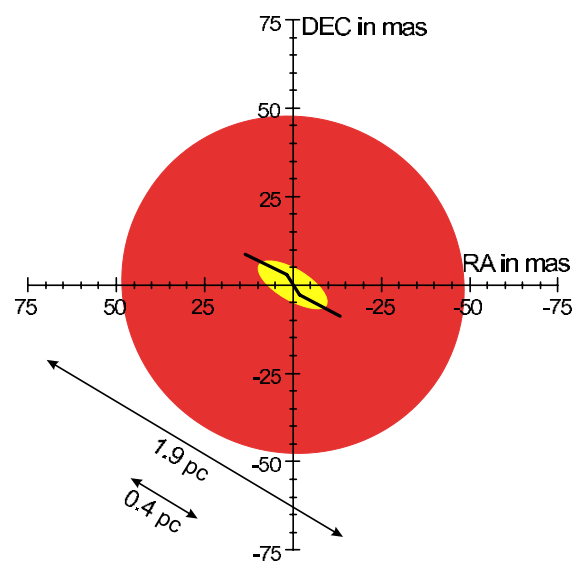

\begin{tabular}{lr}
\hline parameter & best fit value \\
\hline$F W H M_{1}$ & 22.3 \\
$a_{1} / b_{1}$ & 0.39 \\
$T_{1}$ & 387.9 \\
$f_{1}$ & 1.0 \\
$F W H M_{2}$ & 98.4 \\
$a_{2} / b_{2}$ & 0.96 \\
$T_{2}$ & 293.6 \\
$f_{2}$ & 0.5 \\
$\tau_{S i O}$ & 1.2 \\
$\alpha$ & 58.8 \\
$\bar{\chi}^{2}(431$ d.o.f. $)$ & 44.3 \\
\hline
\end{tabular}

Figure 2. Sketch and best fit parameters of the model for Circinus. A highly elongated hot emission region (yellow) is surrounded by an extended, almost round and cooler emission region (brown). At the centre, the locus of $\mathrm{H}_{2} \mathrm{O}$ maser emitters in a disk from Greenhill et al. (2003) is over-plotted. The parameters fitted are: $F W H M$ - full width at half maximum (in mas), $a / b$ - axis ratio, $T$ - temperature (in $\mathrm{K}$ ) and $f$ - filling factor for each of the two components; $\tau_{S i O}$ - optical depth of the silicate absorption and $\alpha$-position angle for both components.

We find a small hot component with a FWHM of 22 mas $(\sim 0.4$ pc at the distance of Circinus), which is highly ellipsoidal and has a temperature of $390 \mathrm{~K}$. This component has the same size and position angle as the rotating maser disc found by Greenhill et al. (2003) (see figure 2). The second component is significantly larger, reflecting the fact that a large part of the flux $(\sim 90 \%)$ is resolved with our interferometric setup. The component has a FWHM of 98 mas, which corresponds to 1.9 pc. The component only has a very small ellipticity and a temperature of less than $300 \mathrm{~K}$. It is a grey body with a filling factor of 0.5 . We consider the $390 \mathrm{~K}$ for the inner component as a lower limit for the highest dust temperature and the $300 \mathrm{~K}$ as an upper limit for the cool component. Clearly there is a temperature gradient from the inside to the outside, indicating that the dust is heated by the nuclear source.

\section{Centaurus A}

Centaurus A (NGC 5128) is the closest active galaxy at a distance of only $3.8 \mathrm{Mpc}$ (1 pc $\sim 50$ mas, Rejkuba 2004). It is an elliptical galaxy undergoing late stages of a merger event with a spiral galaxy. Radio interferometry reveals a structure composed of an unresolved core and a jet within the central parsec.

The results of our MIDI observations are summarised in figure 3a, which shows the total flux and the correlated fluxes of the two baseline sets observed. Similar to Circinus, most of the spectral region is affected by the broad silicate absorption feature. Its depth is identical in the correlated and in the total flux, indicating that both the core and the extended component suffer the same extinction. The [NeII] emission line at $12.9 \mu \mathrm{m}$ is clearly detected at the edge of the total spectra, but not in any of the correlated fluxes. The interferometric observations reveal the existence of two components in the inner parsec of Centaurus A: a resolved component, the "disc", and an unresolved "core".

The disc which is most extended along a position angle of $\sim 110^{\circ}$ is roughly oriented perpendicular to the parsec scale radio jet. The decrease in visibility towards longer wavelengths indicates that the extended emission has a spectrum which rises between 9 and $13 \mu \mathrm{m}$ as expected for emission from thermal dust at temperatures $T<300 \mathrm{~K}$. The 

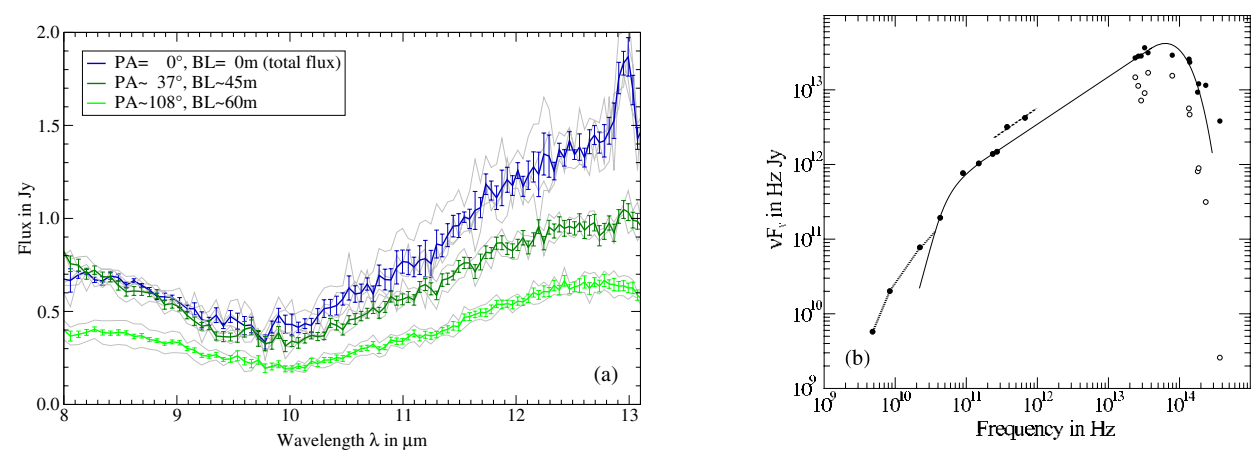

Figure 3. (a) Total flux (blue) and correlated fluxes (green) of Centaurus A. Two baseline sets (consisting of two visibility points each) were observed. (b) Overall spectrum of the core of Centaurus A. Open circles show observed flux values, filled dots are corrected for foreground extinction. The synchrotron spectrum (solid line) shows an optically thin power-law $F \nu \sim \nu^{-0.36}$.

size of the disk is poorly confined by the present observations but needs to be $>30$ mas $(\sim 0.57 \mathrm{pc})$ at $13 \mu \mathrm{m}$, in order to be consistent with our simple two-component model.

In order to understand the nature of the unresolved core emission, we supplemented our mid-infrared photometry by measurements at lower and higher frequencies, using VLBA, millimeter and near infrared data (see figure $3 \mathrm{~b}$ ). From the fact that both the mm-data and the core flux in the MIDI range can be nicely fitted by an optically thin power-law spectrum, we conclude that the core emission is dominated by non-thermal synchrotron radiation. This means that, in Centaurus A, the MIR emission is dominated by an unresolved synchrotron core making up between $60 \%$ (at $13 \mu \mathrm{m}$ ) and $80 \%$ (at $8 \mu \mathrm{m}$ ) of the nuclear MIR flux. The size of this core most likely is $R_{c} \sim 0.01 \mathrm{pc}$, as derived from VLBI observations at $43 \mathrm{GHz}$.

\section{Other objects}

The sensitivity of MIDI is better than initially predicted, so that two fainter AGN, MCG -05-23-016 and Mrk 1239, could be successfully observed. Only one UV point for each object was obtained so far. MCG -05-23-016 is a Seyfert 2 galaxy at a distance of approximately $40 \mathrm{Mpc}$. It also shows a silicate absorption feature. Mrk 1239, is the first Seyfert 1 galaxy observed with MIDI. It is located at a distance of about $90 \mathrm{Mpc}$ and has a flat spectrum in the MIR. The correlated fluxes are consistent with marginally or unresolved objects $\left(F_{\text {cor }} \lesssim F_{\text {tot }}\right)$. The flux level of Mrk 1239 is $400 \mathrm{mJy}$ and that of MCG -05-23-016 only $300 \mathrm{mJy}$.

Considering this success of MIDI, we are confident to observe more AGN soon and to continue our investigations of the already observed targets.

\section{References}

Freeman, K. C. et al. 1977, A\&A, 55, 445

Greenhill, L. J. et al. 2003, ApJ, 590, 162

Jaffe, W. et al. 2004, Nature, 429, 47

Kemper, F., Vriend, W. J. \& Tielens, A. G. G. M. 2004, ApJ, 609, 826

Rejkuba, M. 2004, A\&A, 413, 903

Roche, Patrick, F. et al. 2006, MNRAS, 367, 1689 
Thaisa Storchi-Bergmann: How do you infer that the dust structure you detect in Circinus is rotating?

KonRad Tristram: The $\mathrm{H}_{2} \mathrm{O}$ maser disc observed by Greenhill et at. (2003) has the same orientation and size as the distribution in our model. The maser disc shows a clear Keplerian rotation velocity profile. We conclude hence that the $\mathrm{H}_{2} \mathrm{O}$ masers are embedded in the dust structure and hence the dust is rotating as well.

Felix Mirabel: How does compare your extended dust emission at parsec scales with the very inner image obtained at $2 \mu \mathrm{m}$ with NICMOS on the HST?

KonRAD TRIstram: The high resolution HST images are dominated by the galactical dust-lane on kiloparsec scales in Centaurus A, which is between us and the nucleus. I am not aware of any detection of an extended dust emission in HST images at the nucleus of Centaurus A which would correspond to the emission we detect. 


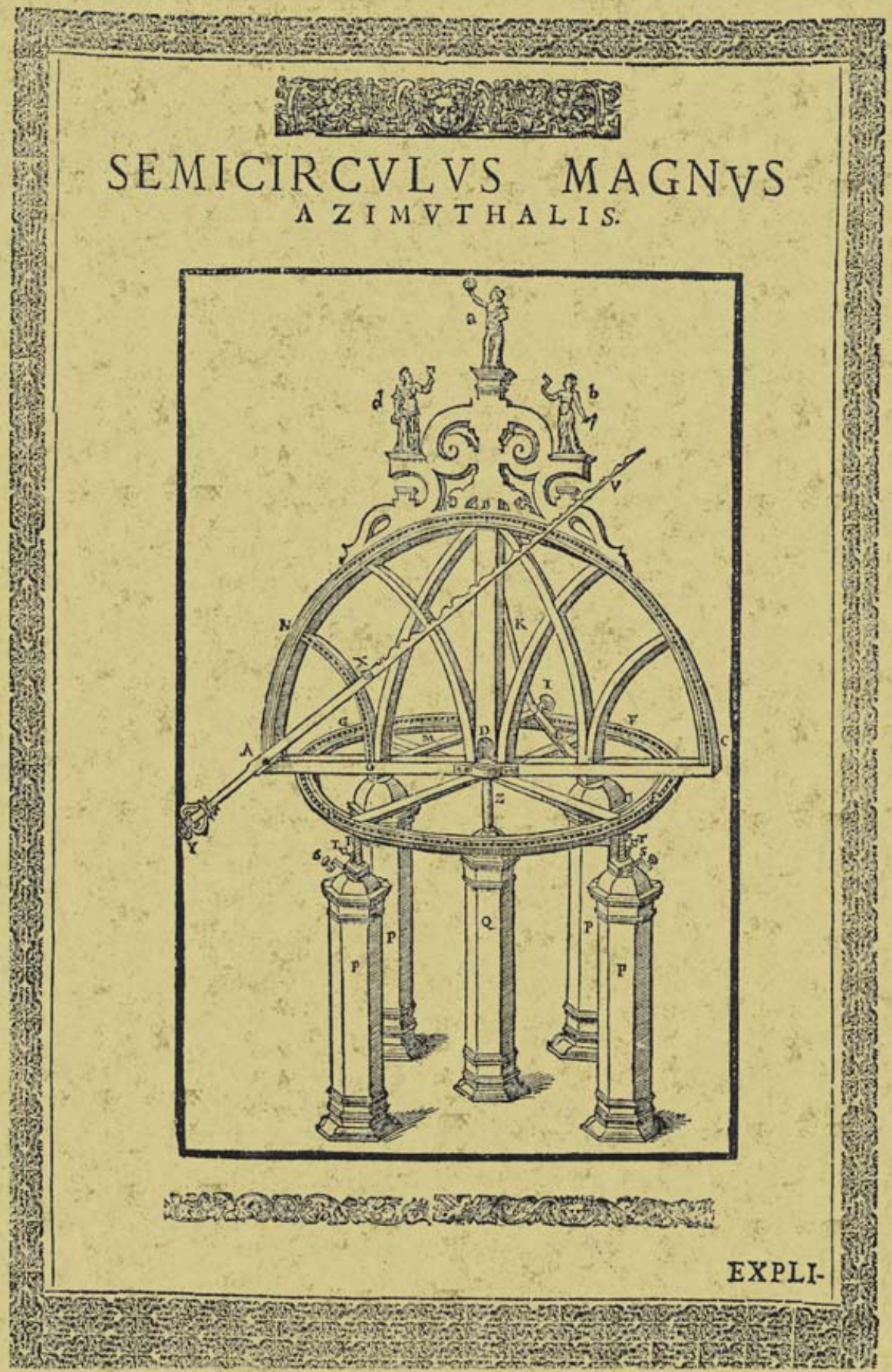

